\title{
Dynamic BOTDA measurements using Brillouin phase-shift
}

\author{
Javier Urricelqui, Ander Zornoza, Mikel Sagues, Alayn Loayssa* \\ Universidad Pública de Navarra, Campus de Arrosadia 31006, Spain
}

\begin{abstract}
We demonstrate a novel dynamic BOTDA sensor based, for the first time to our knowledge, on the use of the Brillouin phase-shift instead of the conventional Brillouin gain. This provides the advantage of measurements that are largely immune to variations in fiber attenuation or changes in pump pulse power. Furthermore, the optical detection deployed can lead to an enhanced precision or measurement time and to the broadening of the measurement range. Proof-of-concept experiments demonstrate $1.66 \mathrm{kHz}$ measurement rate with 1 -m resolution over a 160 -m sensing fiber length.
\end{abstract}

Keywords: Dynamic distributed measurements, Brillouin phase-shift, BOTDA, strain measurements, distributed fiber sensor

\section{INTRODUCTION}

Dynamic measurements of strain are very attractive for structural health monitoring. Brillouin optical time domain analysis (BOTDA) sensors can provide distributed measurements of the strain profile in km-long fibers with high precision and spatial resolution. Nevertheless, a serious drawback of this technique is the need to scan the Brillouin gain spectrum (BGS) by tuning the pump and probe wavelength separation in order to obtain the Brillouin frequency shift (BFS). As a consequence, the measurement time is typically in the order of a few minutes restricting the BOTDA technique to static measurements.

Recently, modifications of the BOTDA scheme for dynamic measurements have been proposed. They are based on tuning the probe wave to the skirt of the BGS so that variations in BFS are translated to changes in the amplitude of the detected probe wave $\mathrm{e}^{1,2,3}$. Key challenges faced by these dynamic BOTDA sensors are: to make measurements tolerant to variations in detected probe wave amplitude that are unrelated to strain changes, such as changes in the attenuation in the sensing fiber or in the pump power; the improvement of the detected signal to noise ratio (SNR), so as to reduce the required averaging; and the increase of the measurement range.

In this work, we demonstrate a novel dynamic BOTDA sensor that is based on the use of the Brillouin phase-shift instead of the conventional Brillouin gain. A phase modulated probe wave is detected and demodulated in the electrical domain so as to obtain an RF phase-shift that is found to be largely independent of the Brillouin peak gain experienced by the probe wave. Therefore, immunity to attenuation variations in the fiber or changes in the power level of the pump pulses is obtained. Furthermore, the optical detection deployed can lead to an enhanced precision, to the broadening of the measurement range and to the enhancement of the measurement time.

\section{FUNDAMENTALS OF THE SYSTEM}

Fig. 1 schematically depicts the fundamentals of the proposed system. A phase-modulated probe wave is injected in one end of an optical fiber while a pump pulse is introduced at the other end. This probe wave interacts with the pump pulse via stimulated Brillouin scattering (SBS) and is directed to the receiver using a circulator. If SBS interaction only affects the upper sideband of the modulation, then the optical field at the input of the photodetector coming from the interaction of pump and probe at a particular location in the fiber, $z$, is given by the following expression:

$$
E(t)=-E_{S B} \exp \left(j 2 \pi\left(v_{0}-f_{R F}\right) t\right)+E_{0} \exp \left(j 2 \pi v_{0} t\right)+E_{S B} \exp \left(j 2 \pi\left(v_{0}+f_{R F}\right) t\right) H_{S B S}\left(v_{0}+f_{R F}, z\right)
$$

where $E_{0}$ and $E_{S B}$ are the complex amplitudes of the optical fields of the carrier and sidebands of the phase-modulated probe wave, $v_{0}$ is the optical frequency of the carrier, $f_{R F}$ is the modulation frequency and $H_{S B S}$ is the complex Brillouin gain spectrum at position $z$, which can be described by:

*alayn.loayssa@unavarra.es; phone 0034 948169840; fax 0034 948169720; unavarra.es 


$$
H_{S B S}(v, z)=\exp \left(g_{B} /\left(1+2 j \frac{v-v_{P}+v_{B}(z)}{\Delta v_{B}}\right)\right)
$$

where $g_{B}$ is the peak gain, $\Delta v_{B}$ is the Brillouin linewidth, $v_{B}$ is the Brillouin frequency shift at position $z$ and $v_{P}$ is the optical frequency of the pump wave. When the pulse duration is near to the acoustic lifetime, the effective interaction spectrum can be approximated by the convolution of this expression with the pulse spectrum.

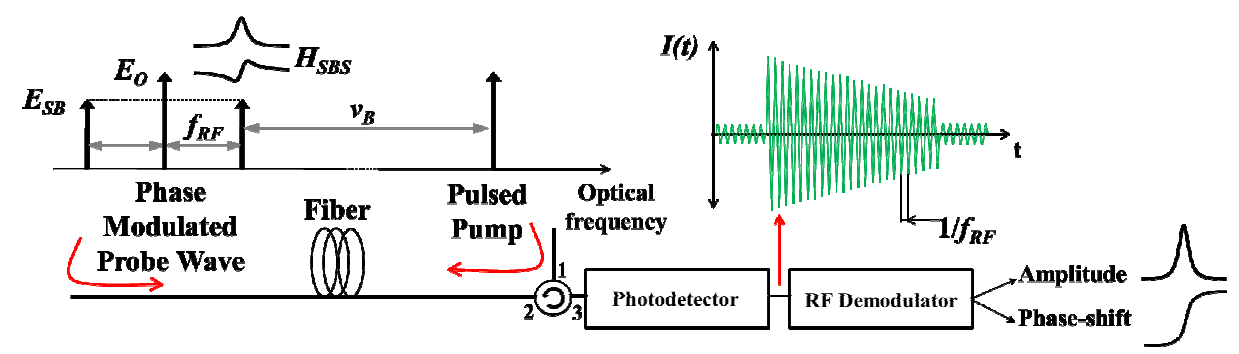

Figure 1. Schematic representation of SBS interaction and the received signal.

The detected RF signal can be written in phasorial form as:

$$
I\left(f_{R F}\right)=R_{D} 2 \sqrt{P_{0} P_{S B}}\left(H_{S B S}\left(v_{0}+f_{R F}, z\right)-1\right) \approx \frac{R_{D} g_{B} \sqrt{P_{0} P_{S B}}}{\sqrt{1+\left(2 \frac{\Delta v}{\Delta v_{B}}\right)^{2}}} \exp \left(-j \arctan \left(2 \frac{\Delta v}{\Delta v_{B}}\right)\right)
$$

where $P_{0}$ and $P_{S B}$ are the optical powers of carrier and modulation sidebands, $R_{D}$ is the responsivity of the photodetector and $\Delta v=v_{0}+f_{R F}-v_{P}+v_{B}$ is the detuning of the interacting sideband from the center of the Brillouin spectrum. The approximation for the last term is obtained assuming a small $g_{B}$, which is the case for BOTDA sensors. Notice that Eq. 3 shows that the detected signal and its SNR can be enhanced simply by increasing the carrier power, while keeping the sideband power low enough to avoid non-local effects ${ }^{4}$. Furthermore, the particular Brillouin peak gain experienced by the probe wave at a given position of the sensing fiber only affects the amplitude of the detected electrical current, but not its phase-shift. This is highlighted in Fig. 2, where the RF phase-shift in our system and the amplitude in a conventional BOTDA are calculated and represented for different values of $g_{B}$. As it is shown, the phase-shift remains largely independent of $g_{B}$ in contrast to the amplitude. This has major implications for dynamic sensing. In conventional dynamic BOTDA, the probe wave is set to a wavelength on the slope of the amplitude spectrum so that any variation in BFS is translated to a change in the detected probe amplitude. Therefore, the measurement is susceptible to errors caused by changes in pump power or attenuation in the fiber. These are highly probable in a structure with dynamic deformation where they would be misinterpreted as strain changes. The technique that we propose is immune to these errors because in our case the probe wave is set to the slope of the detected phase-shift, which is independent of the pump power (via $g_{B}$ ) and of the received probe power.

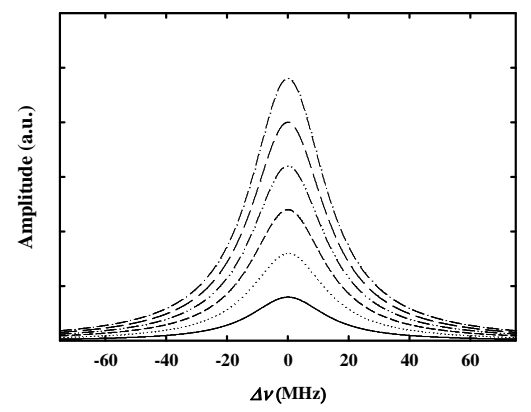

(a)

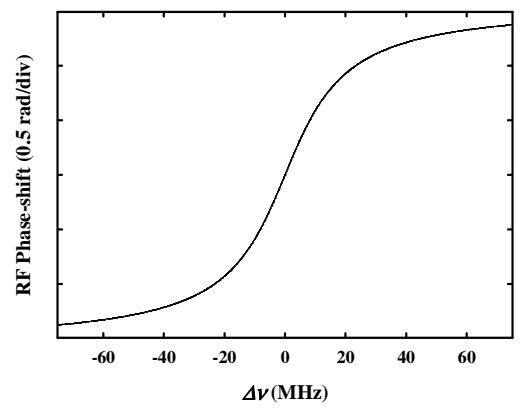

(b)

Figure 2. Calculated (a) amplitude at conventional BOTDA and (b) RF phase-shift of the proposed technique for different values of $g_{B}$. 
Fig. 3 compares the precision of our technique to that of the conventional BOTDA for two cases: equal SNR of $42 \mathrm{~dB}$ for both methods and $10 \mathrm{~dB}$ improvement in SNR for the phase-shift technique due to self-heterodyne detection ${ }^{4}$. This has been numerically calculated by considering Gaussian noise in the received signals of both systems. In addition, just one of the two possible sides of the amplitude spectrum for conventional BOTDA is depicted. Notice that the measurement range for a given minimum precision with the phase-shift technique is larger than that of the conventional BOTDA. For instance, for a minimum precision of $1 \mathrm{MHz}$ (around $20 \mu \varepsilon$ for standard single mode fiber) the range would be $42 \mathrm{MHz}(840 \mu \varepsilon)$ for the phase-shift technique against $26 \mathrm{MHz}$ $(520 \mu \varepsilon)$ for conventional BOTDA. Furthermore, the measurement range increases to $75 \mathrm{MHz}(1500 \mu \varepsilon)$ for the phase-shift technique when the SNR enhancement is assumed.

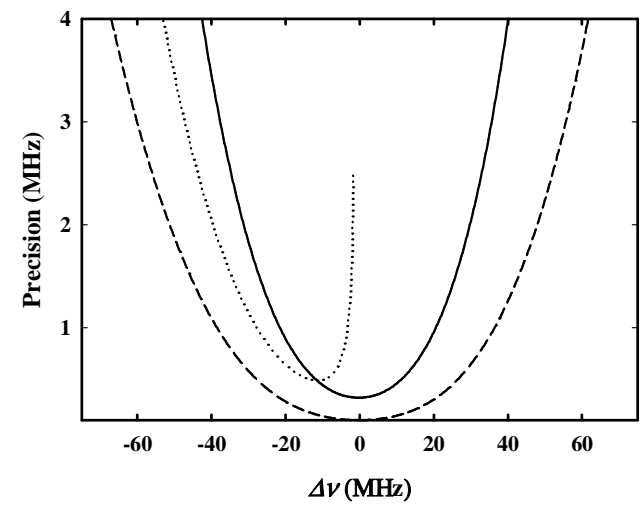

Figure 3. Calculated precision for the conventional BOTDA (dotted line), phase-shift technique (solid line) and phase-shift technique with a $10 \mathrm{~dB}$ SNR enhancement (dashed line).

\section{EXPERIMENTAL RESULTS}

The experimental setup shown in Fig. 4 is assembled in order to demonstrate the system.
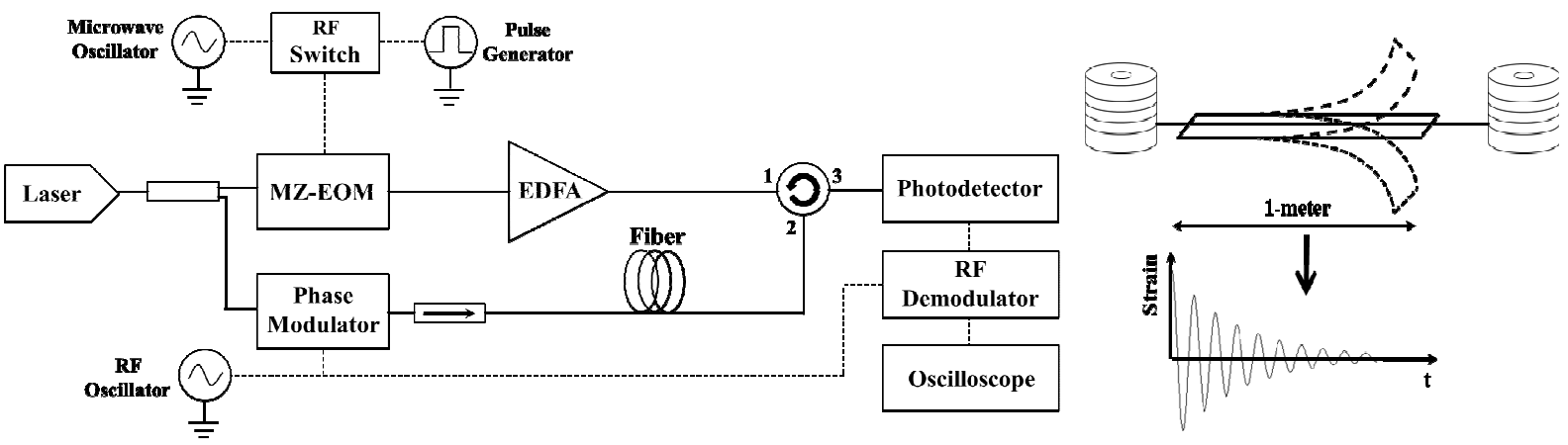

Figure 4. Experimental setup for the phase-shift-based BOTDA dynamic strain measurements.

The output of a laser source is divided in two optical branches with an optical coupler. In the upper branch, the optical pump pulses are formed by the RF pulse-shaping technique ${ }^{5}$ so as to obtain clean and leakage-free pulses. Each generated pulse is $10 \mathrm{~ns}$ long, corresponding to $1 \mathrm{~m}$ spatial resolution. After amplification in an erbium doped fiber amplifier (EDFA), the resulting pump pulses are directed via a circulator to a 160m-long fiber. In the lower branch, the probe wave is generated with an electro-optic phase modulator driven by a $850 \mathrm{MHz}$ RF signal. After interacting with the pump pulses via SBS, the probe signal is directed to a receiver and the resultant RF signal is demodulated. Finally, the BOTDA signal is captured in a digital oscilloscope.

In order to prove that the detected phase-shift is immune to amplitude changes of the BOTDA signal, several spectra were measured for different attenuation values of the pulse and probe signals. Fig. 5a shows that the detected phase-shift remains unaltered while the amplitude (inset) suffers severe attenuation. 


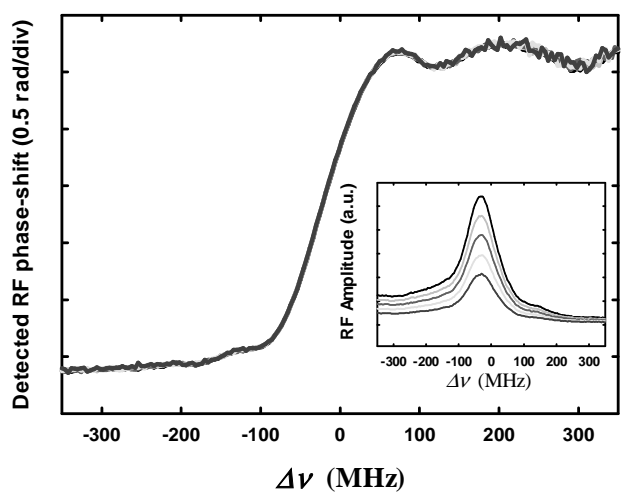

(a)

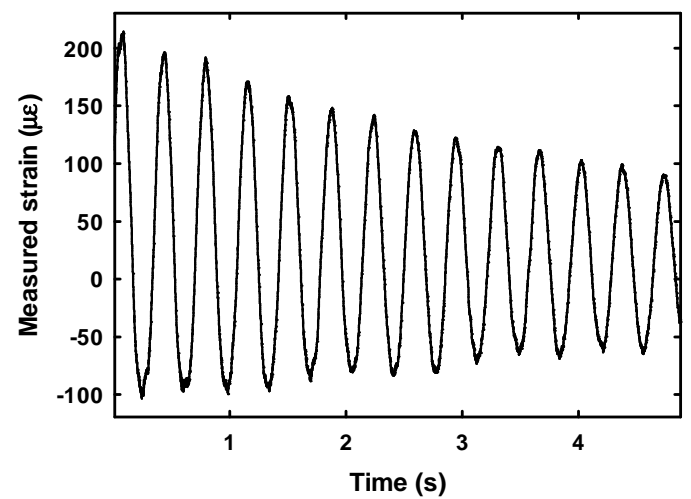

(b)

Figure 5. (a) Scanned phase-shift and amplitude spectra for different attenuation values of the pulse and probe signals, and (b) fast-acquisition measurement of the induced strain at the cantilever beam.

Finally, a 1 meter section of the fiber was affixed by epoxy resin onto the surface of a 1-m cantilever beam. The cantilever beam was made to vibrate so that dynamic measurements of the induced strain could be performed (Fig. $5 \mathrm{~b}$ ). The precision of the sensor was measured to be $20 \mu \varepsilon$ with an averaging of 64 samples. In dynamic distributed strain measurements, the sampling rate only depends on the length of the sensing fiber ${ }^{1}$. Therefore, a potential sampling rate of $625 \mathrm{kHz}$ could be achieved. However, this was limited by the available instrumentation achieving a measurement rate of $1.66 \mathrm{kHz}$.

\section{CONCLUSION}

A novel dynamic Brillouin sensor based on the use of the Brillouin phase-shift has been proposed and demonstrated. The technique relies on the RF phase-shift spectrum, which is proofed to be largely immune to variations of the Brillouin peak gain, attenuation on the fiber, or changes in the power of the pump pulses. Therefore, one of the fundamental drawbacks of the previously reported dynamic BOTDA schemes is overcome. In addition, theoretical calculations show that the technique could lead to a broadening of the measurement range and an enhanced precision. Finally, a proof-of-concept experiment has been performed in order to demonstrate the capabilities of this technique.

\section{ACKNOWLEDGMENTS}

The authors wish to acknowledge financial support from the Spanish Ministerio de Educación y Ciencia through the project TEC2010-20224-C02-01 and from the Universidad Pública de Navarra.

\section{REFERENCES}

[1] Bernini, R., Minardo, A. and Zeni, L., "Dynamic strain measurement in optical fibers by stimulated Brillouin scattering," Optics Letters 34(17), 2613-2615 (2009).

[2] Peled, Y., Motil, A., Yaron, L. and Tur, M., "Distributed and dynamical Brillouin sensing in optical fibers," Proc. SPIE 7753, 775323 (2011).

[3] Cui, Q., Pamukcu, S., Xiao, W. and Pervizpour, M., "Truly Distributed Fiber Vibration Sensor Using Pulse Base BOTDA with Wide Dynamic Range," Photonics Technology Letters IEEE 23(24), 1887-1889 (2011).

[4] Zornoza, A., Sagues, M. and Loayssa, A., "Self-Heterodyne Detection for SNR Improvement and Distributed Phase-Shift Measurements in BOTDA," Journal of Lightwave Technology 30(8), 1066-1072 (2012).

[5] Zornoza, A., Olier, D., Sagues, M. and Loayssa, A., "Brillouin distributed sensor using RF shaping of pump pulses," Measurement Science and Technology 21(9), 094021 (2010). 
Copyright 2012 Society of Photo Optical Instrumentation Engineers. One print or electronic copy may be made for personal use only. Systematic re-production and distribution, duplication of any material in this paper for a fee or for commercial purposes, or modi_cation of the content of the paper are prohibited. 